\title{
Grand Challenges in Analytical Science
}

\author{
Huan-Tsung Chang *t \\ Department of Chemistry, National Taiwan University, Taipei, Taiwan
}

Keywords: analytical science, sensing, screening, miniaturized devices, nanomaterials

\section{INTRODUCTION}

Analytical science is related to the development and application of techniques for detection of analytes, characterization of composites, analysis of samples, and monitoring of chemical and biochemical systems. It has played significant roles in the studies of physical, life, material, environmental, food, medical, and sustainability sciences. In recent years, we have witnessed various techniques for single-cell analysis, screening of circulating tumor cells, viral diagnostics, detection of radioactive substances and explosive compounds, screening and identification of abused drugs, tracking contaminants and chemicals to ensure water quality and food safety, the study of omics, and characterization of synthetic polymers and nanomaterials. For example, various analytical techniques, such as reverse transcription polymerase chain reaction (RT-qPCR), loopmediated amplification (LAMP), and clustered regularly interspaced short palindromic repeats (CRISPR) assays have been applied for sensitive and specific detection of severe acute respiratory syndrome coronavirus 2 (SARS-CoV-2) that causes COVID-19 disease (Huang et al., 2020; Wang et al., 2021). LAMP is attractive because there is no need for temperature cycling and it provides extremely high sensitivity (down to $\mathrm{fM}$ ) with fluorescent, electrochemical, or electroluminescent signal transduction. To minimize the threat of pandemics, vaccines against pathogens such as Zika virus and SARS-CoV-2 have been developed. For quality control and safety of vaccines, many analytical techniques such as sampling, purification, high performance liquid chromatography (HPLC), and gene expression profiling are needed.

To meet the requirement of various studies and needs of society, analytical techniques must be, in general, sensitive, selective, fast, accurate, and simple. The instruments must be cost-effective, easy to operate and maintain, compact (portable ideally), suitable for the analysis of various samples, and available to provide wide dynamic ranges for quantitation of analytes. Analytical techniques are chosen mainly based on the purpose of the study, equipment available, properties of the analyte, and nature of the sample. For example, optical techniques that provide high temporal and spatial resolution are commonly applied for cell tracking. To improve reproducibility, efficiency, and accuracy of the cell studies, the sequential cell images are then subjected to computational object tracking to track cells events over time and to obtain signals from each object. When in-vivo monitoring of drug function is the aim, nonconstructive optical techniques allowing deep penetration from the surface are usually carried out. In this case, materials that can absorb light and generate optical signals like fluorescence in the infrared (IR) or near IR (NIR) regions are suitable. For environmental analysis and forensics, portable and low-cost on-field analytical instruments are ideal.

To provide high specificity and sensitivity for quantitation of various analytes, nanomaterials with high electrochemical activity and conductivity have become more popular in developing electrochemical sensing systems (Wongkaew et al., 2019). Many nanomaterials-based functional electrodes have shown their potential in various fields; for example, fuel cells, removal of contaminants from polluted water, and degradation of toxic chemicals in the air. Nano or micro devices have gained more attention in biological analysis, with advantages of use of small sample 
volume, consumption of extremely low amounts of reagents and solvent, high resolution, and rapidity. Many of them have been applied for differentiation of cancer cells from normal cells, quantitation of circular tumor cells, study of cell-to-cell interaction in the single-cell level, and detection of trace biomarkers (Chen C.-H. et al., 2020). Mass spectrometry (MS) providing high sensitivity and wide linear dynamic ranges makes it suitable for the quantitation of chemicals of interest in environmental and biological samples. Hyphenated techniques such as MS in conjunction with capillary electrophoresis or HPLC have shown potential in various studies such as omics and pharmaceutical analysis, with advantages of automation, rapidity, high resolution, and sensitivity. Nanoscale and microscale electrophoresis systems in conjunction with MS have gained more attention in biological studies, like detection of single cells, bacteria, and viruses. MS integrated with other techniques such as nanospray desorption electrospray ionization plays a strong role in many studies such as molecular profiling and quantification of endogenous species in single cells (Bergmana and Lanekoff, 2017). In the last decade, we have also witnessed more important roles for artificial intelligence (AI) in analytical science.

In the last twenty years, many nanomaterials-based analytical techniques have emerged as powerful analytical tools for various applications (Huang et al., 2007; Lan. et al., 2010; Lee et al., 2014; Roy et al., 2015; Chen et al., 2021). For example, gold nanoparticles and nanoclusters have been used to develop sensitive and selective probes (Chen et al., 2016); $\mathrm{Fe}_{3} \mathrm{O}_{4}$ nanoparticles are useful and efficient adsorbents for the concentration of analytes of interest and for removal of potential interfering species; $\mathrm{TiO}_{2}$ and $\mathrm{HgTe}$ nanoparticles that can absorb laser light efficiently are effective matrixes to enhance laser desorption/ionization efficiencies of various analytes like proteins in MS (Chiang et al., 2011); Ag nanoparticles and Te-Au nanowires as substrates have been used to develop sensitive surface enhancement Raman scattering techniques for detection of chemicals, proteins, DNA, and bacteria (Lin et al., 2011); $\mathrm{SiO}_{2}$ nanoparticles have shown their potential as coating or packing materials to improve separation performance in HPLC, gas chromatography (GC), and capillary electrophoresis. Antibody-functionalized nanomaterials have become popular for the detection of various analytes, bacteria, and cancer cells (Huang et al., 2018). Many selective and sensitive sensing systems using aptamers alone or in conjunction with nanoparticles are specific and sensitive in the quantitation of DNA, proteins, small analytes, and cancer cells (Huang, et al., 2005). Aptamerfunctionalized nanoparticles such as $\mathrm{Au}$ and graphene have been widely used in developing sensitive and selective fluorescent, Raman scattering, electrochemical, and electrochemiluminescent probes for various analytes. Besides aptamers, DNA nanostructures (Li C. et al., 2021) with/ without applying signal amplification strategies such as rolling circle amplification, strand displacement amplification, and signal-mediated amplification (Li et al., 2021) have been demonstrated for the detection of low amounts of analytes like microRNA. Their sensitivity can be further improved when combined with nanomaterials. By taking advantages of the high catalytic activity of nanomaterials such as $\mathrm{Ag}, \mathrm{Ag}-\mathrm{Au}, \mathrm{Pt}$, and $\mathrm{CeO}_{2}$ NPs and carbon dots, many nanozymes have been used to develop sensitive electrochemical, fluorescent, and colorimetric assays for the detection of important analytes such as glucose, $\mathrm{Hg}$ (II), DNA, and proteins (Cheng et al., 2016; Wei et al., 2020).

Although many analytical techniques have been applied successfully for many studies, they sometimes are not ready to meet emergent crises such as a pandemic outbreak, spread of live hazards, release of radioactive substances, and illegal additives in food. To face these challenges, analytical chemists must work closely with experts from different fields such as engineers, clinicians, and/or health practitioners to develop sensitive, selective, low-cost, and portable analytical systems in a short period of time. The analytical systems must also be user-friendly, allow rapid analysis, and only require small amounts of reagent/ solvent. In addition to unexpected issues, significantly different natures in samples, matrixes, analytes, and instruments make it difficult to address all possible challenges that analytical science will face. To highlight the importance of analytical science for fundamental study and to meet societal needs, some challenges as examples will be discussed briefly in the following.

\section{SCREENING OF PATHOGENS}

Development of pathogen sensors is important to minimize life threat and to reduce societal cost due to the outbreak of pathogens such as influenza and SARS-CoV-2. The sensors must be selective, sensitive, low-cost, and easy to operate, which can be utilized in the field, clinics, and ideally homes. Colorimetric, electrochemical, fluorescent, and light scattering techniques are good candidates to be used in the sensing systems. Before developing ideal sensing systems for a new and emerging virus/bacteria-causing pandemic, DNA/RNA sequences of the virus/bacteria, and IgM and IgG antibodies that can be produced days to weeks following exposure to the virus/bacteria must be provided. This usually puts great pressure on the lab to have them ready for the public in a short period of time. Although RT-qPCR tests with a fluorescence detection mode can be developed in a short period of time, they require specialist training to operate and are usually expensive and time-consuming. Immunoassays with colorimetric, fluorescent, or electrochemical detection modes are more suitable for on-field analysis, mainly because of their simplicity in operation. However, poor sensitivity, false results, and matrix interference are sometimes problematic. For this reason, they are usually for screening purposes; the samples with positive results are further subjected to PCR tests or MS analysis. Although nanoparticles functionalized with expensive antibodies have been shown to be effective in enhancing sensitivity, their preparation in a short period of time and at a large scale to be ready for a point-of-care (POC) purpose is unrealistic. Stability and high specificity of the functional nanomaterials toward analytes in complicated samples are another issue. Alternatively, lateral flow assays (LFA), taking advantage of nucleic acid amplification like isothermal recombinase polymerase amplification (RPA), and the specificity of CRISPR and its associated proteins (Cas) have 
become popular for detection of pathogens. For example, a RPACRISPR/Cas9 LFA with a fluorescent detection mode shows sensitive and selective detection of SARS-CoV-2 (Osborn et al., 2021). However, cost, analysis time, false positive and negative results, and complicated operation procedures are concerns before CRISPR/Cas LFA assays with nucleic acid amplification can be ready for POC. To save lives and prevent over run in healthcare facilities, global collaboration to develop simple, sensitive, cost-effective, and accurate pathogen diagnostic tests is needed. It will be more efficient without political interference, which is also a challenge sometimes.

\section{FOOD SAFETY}

Many analytical techniques such as chromatography or electrophoresis in conjunction with MS, UV-vis absorption, and/or fluorescence have been commonly applied to ensure food safety. For samples containing extremely low amounts of analytes and/or complicated matrixes, sample pretreatment using liquid-liquid extraction, solid-phase extraction, solid-phase microextraction, and solvent trapping are usually applied. However, low recovery efficiency and low extraction percentage are usually problematic. On the other hand, it is very challenging to detect trace analytes from small amounts of complicated samples. Sometimes, multiple chromatography separation (e.g., reversible-phase HPLC and ion-exchange HPLC) with an extremely sensitive MS system may be possible. However, such a system may not work successfully for the analysis of samples contaminated with commercial insecticides and pesticides that contain many active chemicals, each at a concentration under their legal limit. To ensure food safety, it is important to utilize chromatography-MS systems, allowing detection of all active chemicals. It is even more challenging when the trace analyte is unknown. In addition, most of the HPLC-MS techniques are developed for the analysis of organic compounds; techniques allowing detection of different types of analytes such as large-molecular-weight surfactants and plastic microbeads are urgent. Because of the high cost of the systems and their maintenance, and the requirement of experienced operators, they are not suitable for use at home.

Low-cost sensing systems such as enzyme-linked immunosorbent assays in an LFA or array configuration are common and more affordable for screening of potential food contaminants such as pesticides and bacteria. Common detection modes include colorimetry, electrochemistry, fluorescence, and Raman scattering spectroscopy. They may provide enough sensitivity for detection of food contaminants, but problems are usually raised when the matrix is complex. To further improve sensitivity, nanomaterials such as gold nanoparticles, silver nanoparticles, and gold nanoclusters have been used to ensure food safety (Han et al., 2020; Tao et al., 2020). When new or unknown pesticides, insecticides, or bacterial variants are present, standard approaches usually do not work. It is not an easy task to develop sensitive and selective sensing systems to meet the emergent needs. Array sensing systems are required for detection of several contaminants present in food samples, it however is difficult to obtain both sensitive and selective array probes. It is also urgent to develop techniques for sensitive and specific detection of food contaminants such as parasites, viruses, and fungi, mainly because of the few commercial probes available and the lack of standard approaches.

\section{HEATH MONITORING}

Many sensing kits for health monitoring have been developed for use in the home, including glucose meters and pregnancy test kits. With advances in wearable technology, many wearable devices providing important body information like blood glucose concentration, blood oxygen level, blood pressure, and heart rhythms have been sold in the market. Electrochemical sensors are mostly found in wearable devices, mainly because of their advantages of miniaturization, low cost, fast response, and high sensitivity and specificity (Hernández-Rodríguez et al., 2021). In addition, electrodes can be added to various substrates such as plastic, textile thread, glass, ceramic, or skin. Fabrication of microelectrodes on substrates with good quality in a large scale remain a challenge, although photolithographic technologies are available. To meet healthcare needs, the microelectrode sensors must provide high selectivity and sensitivity for early detection of diseases like cancer or screening of pathogens, and continuous monitoring of treatment effectiveness. To achieve this goal, microelectrodes must be functionalized with enzymes or nano materials with enzyme-like properties. Preparation of cost-effective and stable functional microelectrodes with enzymes is sometimes problematic. On the other hand, the catalytic activity and specificity of nanomaterials used for fabrication of functionalized microelectrodes is usually lower than that of nature enzymes although many candidates such as $\mathrm{Au}, \mathrm{BiO}$, $\mathrm{CuO}$, and $\mathrm{Au}$ alloy nanoparticles have shown their potential (Lien et al., 2018). To minimize matrix interference, sample pretreatment and separation using microfluidic or nanofluidic designs in wearable devices are usually required when handling biofluids. It is a challenge for the fabrication of such multiple functional wearable devices. Integration of wireless and internet of things (IoT) devices that allow patients to transfer data to clinicians/doctors for real-time decision-making is another challenge. Issues such as long-term stability and comfortability of wearable devices must also be considered carefully. Accuracy and reliability of the results provided by the microelectronic sensors must be checked and addressed frequently. Wearable devices for healthcare are definitely still not mature enough, worldwide collaboration among researchers from fields of chemistry, biochemistry, engineering, and medicine is needed.

\section{SCREENING OF ABUSED DRUGS}

Illicit drugs such as cocaine, heroin, ketamine, and marijuana have caused serious societal problems around the world. Some anxiolytic (hypnotic) drugs such as alprazolam and clonazepam for treating sleep disorders have been used for drug-facilitated 
sexual assault. Many new psychoactive substances (NPS) such as synthetic cannabinoids and cathinones have become a more serious problem, mainly because they are more potent and dangerous than their corresponding substances extracted from plants. In the last twenty years, more than 190 synthetic cannabinoids have been found in the market according to a report from the European Monitoring Center for Drugs and Drug Addiction. Although hyphenated chromatography-MS systems such as GC-MS, GC-MS/MS, and HPLC-MS/MS are powerful for the identification of abused drugs (Pellegrini et al., 2020), numerous suspected samples collected by law enforcement officers have put great pressure on the labs. When samples containing several new abused drugs, additives, and complicated matrices, the standard approaches may not be able to provide accurate results in a short period of time. To reduce the burden put on the lab, commercial sensing kits such as immunoassays or chemical approaches with fluorescent, electrochemical, or colorimetric detection modes have been used for screening of the above addressed commonly abused drugs. However, immunoassays with high specificity and sensitivity for synthetic NPs are few, mainly because antibodies with high affinity towards NPs are unavailable. It is even more difficult to engineer antibodies with the capability to differentiate NPs and their derivatives. To prevent the use of antibodies and enzymes, functional nanomaterials have been used for screening illicit drugs (Liu and Lu, 2006; Chen J. et al., 2020). For example, based on analyte-induced aggregation of $\mathrm{Au}$ nanoparticles or disassembly of $\mathrm{Au}$ particle aggregates, colorimetric approaches have been shown to be sensitive and selective for the detection of illicit drugs like cocaine. Alternatively, Ag nanoclusters, carbon dots, upconversion nanoparticles, and quantum dots have been used to develop fluorescent probes for the detection of illicit drugs, including codeine, cocaine, and ketamine, based on analyteinduced changes in their fluorescence. Carbon dots that are easily prepared from carbon precursors have been used for the screening of cathinones and date rape drugs (Yen et al., 2019; Yen et al., 2020). Interference from colorful sample matrices is usually less serious when applying fluorescence over colorimetric approaches. When the screening of multiple illicit drugs is needed, array sensing systems with various functional nanomaterials is required. Because it is difficult for each of the nanomaterial-based probes to have high affinity toward a specific illicit drug, a sensing system with machine learning is suggested to improve the performance of multiple drug screening. In this case, the selection of nanomaterials and determination of the detection threshold (machine learning) for each sensing element are challenges. It is also tough when the suspected samples contain a complicated matrix.

Recently, compact Raman scattering systems (around USD $50 \mathrm{~K})$ have gained popularity for the screening of abused drugs, but they still face challenges in screening NPs. It is difficult if the suspected samples are not pure and their concentrations are low. In this case, functional nanoparticles like silver nanoparticles as an adsorbent and a surface-enhanced Raman scattering (SERS) substrate are worthy of investigation. Preparation of functional nanomaterials for a large number of illicit drugs is definitely difficult at present. In addition, reproducible and accurate results provided by SERS have to be addressed carefully. It is difficult for most research labs to obtain illicit drugs, and thus it requires close collaboration among researchers, law enforcement officers, and administrators.

\section{CHARACTERIZATION OF NANOMATERIALS}

Nanomaterials have shown many advanced applications in various fields such as fuel cells, solar cells, catalysts, nanozymes, and sensing (Wu et al., 2020; Xu et al., 2020; Lin et al., 2021). Although many approaches have been used for preparation of various nanomaterials, it is difficult to obtain high-quality nanoparticles (e.g., slight variation in their sizes, surface ligands, and morphologies). Techniques, including UV-vis absorption, fluorescence, Fourier transform infrared, electrochemistry, laser light scattering, Raman scattering, X-ray photoelectron spectroscopy, MS, transmission electron microscopy (TEM), and scanning electron microscopy are often applied to characterize nanomaterials (Mourdikoudis et al., 2018). However, it is difficult to differentiate the oxidation states of some elements like $\mathrm{Cu}(\mathrm{I})$ and $\mathrm{Cu}(\mathrm{II})$. Techniques allowing direct determination of the amounts of surface ligands on each particle are still required. Such information is not only important for understanding the chemical and physical properties of nanomaterials, but also for designing functional nanomaterials to have optimal properties like recognition ability, stability, and fluorescence quantum yield. Although TEM allows for the recording of highquality large-size images of nanomaterials, it does not provide clear images for sub-nanometer nanomaterials such as DNA-Ag nanoclusters. It is also difficult to obtain high-quality images for small sizes of low-contrast nanomaterials like carbon dots. Dark-field light scattering techniques can provide clear images of nanomaterials with sizes above $10 \mathrm{~nm}$, but it is difficult to obtain smaller size of nanomaterials, especially those with a small cross section. Thus, optical techniques allowing recording of images of nanomaterials with sizes smaller than $10 \mathrm{~nm}$ are demanded. Although MS with electrospray ionization or laser desorption ionization and induced coupled plasma-MS have been used to provide important information about protein-templated $\mathrm{Au}$ nanoclusters and DNA-Ag nanoclusters, determination of their chemical structures and accurate molecular weight still remain a challenge. Techniques providing accurate and detailed information about the size, morphology, surface defect, surface ligands, and oxidation state of carbon-based nanomaterials such as graphene and carbon dots are another challenge although TEM, SERS, absorption, and/or fluorescence have been commonly applied for their characterization. The information is extremely important to explain their chemical and optical properties. To take full advantage of nanotechnology for various applications, analytical techniques providing more detailed surface and junction information of nanomaterials are highly demanded. 


\section{PROSPECTIVE}

Specificity, sensitivity, accuracy, reproducibility, highthroughput, automation, miniaturization, simplicity, and costeffectiveness will still be key requirements for developing new analytical techniques to meet the needs of scientific research, industrial development, societal events, and healthcare. Nanomaterials will remain important in developing sensitive and selective analytical approaches for the screening of various targets, especially pathogens, environmental, and biological contaminants, hazards, abused drugs, and biological markers associated with diseases. We can also foresee that more nanozymes combined with signal amplification will be used to replace expensive enzymes in developing sensitive and specific assays for food safety, water quality control, and disease diagnostics. More advanced hyphenated techniques of MS with separation techniques such as nano HPLC and nanofluidic/microfluidic devices, and various detection modes will be developed for the study of biological functions and analysis of complicated samples. Imaging techniques of MS combined

\section{REFERENCES}

Bergman, H.-M., and Lanekoff, I. (2017). Profiling and Quantifying Endogenous Molecules in Single Cells Using Nano-DESI MS. Analyst 142, 3639-3647. doi:10.1039/c7an00885f

Chen, C.-H., Wang, C.-C., Ko, P.-Y., and Chen, Y.-L. (2020a). Nanomaterial-based Adsorbents and Optical Sensors for Illicit Drug Analysis. J. Food Drug Anal. 28, 655-677. doi:10.38212/2224-6614.1137

Chen, J., Liu, C.-Y., Wang, X., Sweet, E., Liu, N., Gong, X., et al. (2020b). 3D Printed Microfluidic Devices for Circulating Tumor Cells (CTCs) Isolation. Biosens. Bioelectron. 150, 111900. doi:10.1016/j.bios.2019.111900

Chen, P.-C., Periasamy, A. P., Harroun, S. G., Wu, W.-P., and Chang, H.-T. (2016). Photoluminescence Sensing Systems Based on Copper, Gold and Silver Nanomaterials. Coord. Chem. Rev. 320-321, 129-138. doi:10.1016/j.ccr.2015.12.002

Chen, W., Yao, Y., Chen, T., Shen, W., Tang, S., and Lee, H. K. (2021). Application of Smartphone-Based Spectroscopy to Biosample Analysis: A Review. Biosens. Bioelectron. 172, 112788. doi:10.1016/j.bios.2020.112788

Cheng, H., Zhang, L., He, J., Guo, W., Zhou, Z., Zhang, X., et al. (2016). Integrated Nanozymes with Nanoscale Proximity for In Vivo Neurochemical Monitoring in Living Brains. Anal. Chem. 88 (10), 5489-5497. doi:10.1021/ acs.analchem.5b0392610.1021/acs.analchem.6b00975

Chiang, C.-K., Chen, W.-T., and Chang, H.-T. (2011). Nanoparticle-based Mass Spectrometry for the Analysis of Biomolecules. Chem. Soc. Rev. 40, 1269-1281. doi:10.1039/c0cs00050g

Han, A., Hao, S., Yang, Y., Li, X., Luo, X., Fang, G., et al. (2020). Perspective on Recent Developments of Nanomaterial Based Fluorescent Sensors: Applications in Safety and Quality Control of Food and Beverages. J. Food Drug Anal. 28, 487-508. doi:10.38212/2224-6614.1270

Hernández-Rodríguez, J. F., Rojas, D., and Escarpa, A. (2021). Electrochemical Sensing Directions for Next-Generation Healthcare: Trends, Challenges, and Frontiers. Anal. Chem. 93 (1), 167-183. doi:10.1021/acs.analchem.0c04378

Huang, C.-C., Huang, Y.-F., Cao, Z., Tan, W., and Chang, H.-T. (2005). Aptamermodified Gold Nanoparticles for Colorimetric Determination of PlateletDerived Growth Factors and Their Receptors. Anal. Chem. 77, 5735-5741. doi:10.1021/ac050957q

Huang, C.-C., Yang, Z., Lee, K.-H., and Chang, H.-T. (2007). Synthesis of Highly Fluorescent Gold Nanoparticles for Sensing Mercury(II). Angew. Chem. 119, 6948-6952. doi:10.1002/ange.200700803

Huang, J.-Y., Lin, H.-T., Chen, T.-H., Chen, C.-A., Chang, H.-T., and Chen, C.-F. (2018). Signal Amplified Gold Nanoparticles for Cancer Diagnosis on PaperBased Analytical Devicesfied Gold Nanoparticles for Cancer Diagnosis on with optical detection systems such as fluorescence and SERS that can provide high temporal and spatial resolution remain challenges for single-cell analysis, tissue mapping, and characterization of nanomaterials. It is expected that AI will remain important in developing automatic analytical systems that allow rapid analysis of samples and processing the obtained data for various studies such as omics, diagnostics, and drug screening. Although many analytical systems are available, new analytical approaches are still required to support fundamental research and to respond to emergent needs quickly and accurately. For example, analytical techniques must be ready for accurate and rapid screening and identification of pathogens immediately to minimize the global impact of the next pandemic.

\section{AUTHOR CONTRIBUTIONS}

The author confirms being the sole contributor of this work and has approved it for publication.

Paper-Based Analytical Devices. ACS Sens. 3, 174-182. doi:10.1021/ acssensors.7b00823

Huang, Z., Tian, D., Liu, Y., Lin, Z., Lyon, C. J., Lai, W., et al. (2020). Ultra-sensitive and High-Throughput CRISPR-P Owered COVID-19 Diagnosis. Biosens. Bioelectron. 164, 112316. doi:10.1016/j.bios.2020.112316

Lan, G.-Y., Huang, C.-C., and Chang, H.-T. (2010). Silver Nanoclusters as Fluorescent Probes for Selective and Sensitive Detection of Copper Ions. Chem. Commun. 46, 1257-1259. doi:10.1039/b920783j

Lee, J. D., Cang, J., Chen, Y.-C., Chen, W.-Y., Ou, C.-M., and Chang, H.-T. (2014). Detection of Adenosine $5^{\prime}$-triphosphate by Fluorescence Variation of Oligonucleotide-Templated Silver Nanoclusters. Biosens. Bioelectron. 58, 266-271. doi:10.1016/j.bios.2014.02.068

Li, C., Luo, S., Wang, J., Shen, Z., and Wu, Z.-S. (2021a). Nuclease-resistant Signaling Nanostructures Made Entirely of DNA Oligonucleotides. Nanoscale 13, 7034-7051. doi:10.1039/d1nr00197c

Li, F., Li, G., Cao, S., Liu, B., Ren, X., Kang, N., et al. (2021b). Target-triggered Entropy-Driven Amplification System-Templated Silver Nanoclusters for Multiplexed microRNA Analysis. Biosens. Bioelectron. 172, 112757. doi:10.1016/j.bios.2020.112757

Lien, C.-W., Unnikrishnan, B., Harroun, S. G., Wang, C.-M., Chang, J.-Y., Chang, H.-T., et al. (2018). Visual Detection of Cyanide Ions by Membrane-Based Nanozyme Assay. Biosens. Bioelectron. 102, 510-517. doi:10.1016/ j.bios.2017.11.063

Lin, Y.-S., Lin, Y.-F., Nain, A., Huang, Y.-F., and Chang, H.-T. (2021). A Critical Review of Copper Nanoclusters for Monitoring of Water Quality. Sensors Actuators Rep. 3, 100026. doi:10.1016/j.snr.2021.100026

Lin, Z.-H., Chen, I.-C., and Chang, H.-T. (2011). Detection of Human Serum Albumin through Surface-Enhanced Raman Scattering Using Gold "pearl Necklace" Nanomaterials as Substrates. Chem. Commun. 47, 7116-7118. doi:10.1039/c1cc11818h

Liu, J., and Lu, Y. (2006). Fast Colorimetric Sensing of Adenosine and Cocaine Based on a General Sensor Design Involving Aptamers and Nanoparticles. Angew. Chem. Int. Ed. 45, 90-94. doi:10.1002/anie.200502589

Mourdikoudis, S., Pallares, R. M., and Thanh, N. T. K. (2018). Characterization Techniques for Nanoparticles: Comparison and Complementarity upon Studying Nanoparticle Properties. Nanoscale 10, 12871-12934. doi:10.1039/ C8NR02278

Osborn, M. J., Bhardwaj, A., Bingea, S. P., Knipping, F., Feser, C. J., Lees, C. J., et al. (2021). CRISPR/Cas9-based Lateral Flow and Fluorescence Diagnostics. Bioengineering 8, 23. doi:10.3390/bioengineering8020023

Pellegrini, M., Marchei, E., Papaseit, E., Farré, M., and Zaami, S. (2020). UHPLCHRMS and GC-MS Screening of a Selection of Synthetic Cannabinoids and 
Metabolites in Urine of Consumers. Medicina 56, 408, doi:10.3390/ medicina56080408

Roy, P., Chen, P.-C., Periasamy, A. P., Chen, Y.-N., and Chang, H.-T. (2015). Photoluminescent Carbon Nanodots: Synthesis, Physicochemical Properties and Analytical Applications. Mater. Today 18, 447-458. doi:10.1016/j.mattod.2015.04.005

Tao, X., Peng, Y., and Liu, J. (2020). Nanomaterial-based Fluorescent Biosensor for Veterinary Drug Detection in Foods. J. Food Drug Anal. 28, 576-595. doi:10.38212/2224-6614.1267

Wang, R., Qian, C., Pang, Y., Li, M., Yang, Y., Ma, H., et al. (2021). opvCRISPR: One-Pot Visual RT-LAMP-CRISPR Platform for SARS-Cov-2 Detection. Biosens. Bioelectron. 172, 112766. doi:10.1016/j.bios.2020.112766

Wei, S.-C., Lin, Y.-W., and Chang, H.-T. (2020). Carbon Dots as Artificial Peroxidases for Analytical Applications. J. Food Drug Anal. 28, 559-575. doi:10.38212/2224-6614.1090

Wongkaew, N., Simsek, M., Griesche, C., and Baeumner, A. J. (2019). Functional Nanomaterials and Nanostructures Enhancing Electrochemical Biosensors and Lab-On-A-Chip Performances: Recent Progress, Applications, and Future Perspective. Chem. Rev. 119, 120-194. doi:10.1021/acs.chemrev.8b00172

Wu, C.-W., Unnikrishnan, B., Periasamy, A. P., Chen, I.-W. P., Tseng, Y.-T., Yang, Y.-Y., et al. (2020). Importance of Cobalt-Doping for the Preparation of Hollow $\mathrm{CuBr} / \mathrm{Co} @ \mathrm{CuO}$ Nanocorals on Copper Foils with Enhanced Electrocatalytic Activity and Stability for Oxygen Evolution Reaction. ACS Sust. Chem. Eng. 8, 9794-9802. doi:10.1021/acssuschemeng.0c02309

Xu, D., Lin, Q., and Chang, H. T. (2020). Recent Advances and Sensing Applications of Carbon Dots. Small Methods 4, 1900387. doi:10.1002/smtd.201900387
Yen, Y.-T., Lin, Y.-S., Chen, T.-H., Chyueh, S.-C., and Chang, H.-T. (2020). A Carbon-Dot Sensing Probe for Screening of Date Rape Drugs: nitro-containing Benzodiazepines. Sensors Actuators B: Chem. 305, 127441. doi:10.1016/ j.snb.2019.127441

Yen, Y.-T., Lin, Y.-S., Chen, T.-Y., Chyueh, S.-C., and Chang, H.-T. (2019). Carbon Dots Functionalized Papers for High-Throughput Sensing of 4chloroethcathinone and its Analogues in Crime Sites. R. Soc. Open Sci. 6, 191017. doi:10.1098/rsos.191017

Conflict of Interest: The author declares that the research was conducted in the absence of any commercial or financial relationships that could be construed as a potential conflict of interest.

Publisher's Note: All claims expressed in this article are solely those of the authors and do not necessarily represent those of their affiliated organizations, or those of the publisher, the editors and the reviewers. Any product that may be evaluated in this article, or claim that may be made by its manufacturer, is not guaranteed or endorsed by the publisher.

Copyright $(0) 2021$ Chang. This is an open-access article distributed under the terms of the Creative Commons Attribution License (CC BY). The use, distribution or reproduction in other forums is permitted, provided the original author(s) and the copyright owner(s) are credited and that the original publication in this journal is cited, in accordance with accepted academic practice. No use, distribution or reproduction is permitted which does not comply with these terms. 